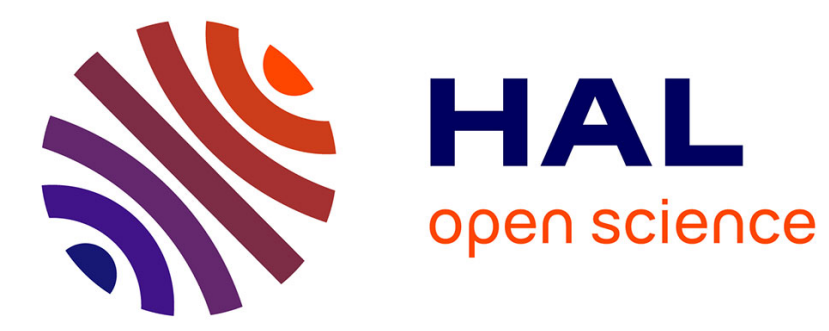

\title{
Exploring Duopoly Markets with Conjectural Variations
}

Ludovic A. Julien, Olivier Musy, Aurélien Saidi

\section{To cite this version:}

Ludovic A. Julien, Olivier Musy, Aurélien Saidi. Exploring Duopoly Markets with Conjectural Variations. Journal of Economic Education, 2014, 45 (4), pp.330-346. 10.1080/00220485.2014.946545 . hal-01385953

\section{HAL Id: hal-01385953 \\ https://hal.parisnanterre.fr/hal-01385953}

Submitted on 21 Nov 2017

HAL is a multi-disciplinary open access archive for the deposit and dissemination of scientific research documents, whether they are published or not. The documents may come from teaching and research institutions in France or abroad, or from public or private research centers.
L'archive ouverte pluridisciplinaire HAL, est destinée au dépôt et à la diffusion de documents scientifiques de niveau recherche, publiés ou non, émanant des établissements d'enseignement et de recherche français ou étrangers, des laboratoires publics ou privés. 


\title{
Exploring Duopoly Markets with Conjectural Variations
}

\author{
Ludovic A. Julien, Olivier Musy, Aurélien W. Saïdi
}

In modern microeconomic textbooks, large portions are usually devoted to the introduction of industrial organization (IO) topics. One of the main concerns, according to Einat and Levin $(2010,146)$, is the analysis of "the structure of industries in the economy and the behavior of firms and individuals in these industries." More precisely, "a long-standing and central problem in Industrial Organization is the extent to which market outcomes reflect the exercise of market power or some form of implicit or explicit collusion" (Einat and Levin 2010, 151). The aim of such textbooks is then to cover some simple alternative representations of firm behavior in order to explain the differences in market power empirically observed across sectors or industries.

The generic approach of market structure commonly used in textbooks consists of the succession of several steps of growing complexity. ${ }^{1}$ It begins with perfect competition, frequently presented as the coexistence of a large number of price-taking firms. Imperfect competition is 
introduced in a second step, usually starting with the study of the monopoly. The economy thus reduces to a single price-making firm. Whether in the competitive or in the monopolistic structure, the firm behaves in isolation. By contrast, after introducing a second price-making firm in a third step, the duopoly structures so-obtained necessarily display strategic interactions. The two firms are given several options to interact strategically: They can play cooperatively or not, either in a simultaneous or a sequential way.

The studies on market structure alternate analytical reasoning with graphical demonstrations. From an analytical viewpoint, students are asked to perform as many calculations and to handle as many problem-solving strategies as the number of market structures under study. They usually experience problems in making the connection between the various frameworks that may be thought of as being relatively independent from each other, especially when strategic interactions are introduced. From a graphical viewpoint, the planes used to frame the market structure can create confusion. For instance, the monopoly equilibrium is framed in a price/quantity space while the various duopoly equilibria are framed in the space of quantities. The graphical arguments also differ from one framework to the other: The Cournot-Nash equilibrium emerges from the intersection of the best-response functions of the two firms while the Stackelberg (1934/2011) equilibrium is pictured through the convergence of isoprofit curves toward the best-response function of the follower. Finally, collusion is represented by the isoprofit curves of both firms. Thus, even if they share a common set of assumptions (especially partial equilibrium, linear demand, and constant marginal cost), these alternative configurations display a deep diversity in their storytelling and analytical or graphical resolutions. This feature has already been pointed out as a potential source of pedagogical issues, encouraging economists to propose a unified framework. To this end, Fulton (1997) developed an integrated graphical presentation of different oligopoly configurations, based on the duality between the marginal cost/revenue trade-off on one hand and an analysis in terms of reaction curves on the other. In a similar way, D' Agata (2010) proposed a reinterpretation of the Cournot-Nash equilibrium in the price/quantity space, usually dedicated to the monopoly. ${ }^{2}$

Another key issue for the introductory studies on market structure is the lack of connection with the concept of market power. The associated measures, such as the Lerner or the Herfindahl indexes, are only tackled in IO textbooks (e.g., Cabral 2000; Tirole 1988). When they are discussed, they only appear in a separated section, unrelated to the market structure analysis. As a result, while many arguments presented in the market structure analysis are based on graphics, these graphics are no longer used to encompass the market power measures, creating an artificial heterogeneity in the presentation.

In this article, we propose to study the various market structures (including monopoly and perfect competition as polar cases) by using the concept of conjectural variations in a two-firm framework. Firms are assumed to form conjectures about their rival's reaction when changing their own strategy; these conjectures influence their optimal decision. The bilateral interaction is captured by a couple of individual conjectural best-response functions. Modeling conjectures helps to fix the issues evoked previously by providing a unified storytelling with a single comprehensive problem-solving procedure. The equilibrium outcome can be computed once for all and parameterized according to the conjectural variations. Then, the different market structures can be replicated by assigning a specific value to the conjectures and pictured in a same figure capturing the alternative firm behaviors - whether competitive, collusive, or oligopolistic. In addition, our framework establishes a connection with the Lerner and the Herfindahl indexes, measuring respectively the market power and the market concentration of firms. Each combination of the 
two indexes corresponds to a specific pair of conjectures, thus to a unique conjectural equilibrium and market structure. Graphically, the equilibria can be deduced either from the conjectural best-response functions or from the indexes. Equivalently, they can be ranked according to the magnitude of their market power and market concentration, which depend on the value of the conjectures. By contrast with many existing unified approaches, our framework is also easily able to handle asymmetric behaviors (when firms form different conjectures).

The use of conjectural variations to represent the various market structures is not new as such. It has been proposed for theoretical and empirical research by Bresnahan (1981) and Dixit (1986), and as a flexible pedagogical tool in several textbooks of microeconomics (Varian 1993; Carlton and Perloff 1994; Estrin, Laidler, and Dietrich 2008) or applied IO (Perloff, Karp, and Golan 2007). However, these authors restrict their analyses to a few cases only. We extend the framework to Stackelberg's (1934/2011) duopoly and to more "exotic" configurations such as the double leader case, originally suggested by Bowley (1924) and presented in some textbooks (see, e.g., Picard 2007). Furthermore, they do not provide any graphical presentation of the equilibria and do not make any connection with the market power indexes.

While the use of conjectural variations encountered some criticisms regarding its theoretical foundations, it can serve as a useful pedagogical device. It notably enables one to capture in a tractable and common framework any possible market structure studied in textbooks, using a unified method of resolution. ${ }^{3}$ Hence, once the conjectural equilibrium is determined, it is possible to deduce any market outcome. In addition, as mentioned previously, the graphical representation of all equilibria may be easily handled in the sole quantity space. In this context, each market outcome is given by the intersection of conjectural best-response functions. Therefore, for our purpose, the use of conjectural variations can represent a shortcut to analyze market interactions (McMillan 1986). This approach can be used both as a synthesis of the chapters devoted to oligopoly and afterward, as a lightening on the diversity of imperfect competition. This synthesis also can be considered as a transition from theoretical models to empirical applications because the conjectural variations are often used as a preliminary framework to estimate aggregate market power (see Perloff, Karp, and Golan 2007, ch. 3, for a presentation).

This article is organized as follows. In the next section, we present the model setup and define the set of notions of the conjectural approach. We make use of these tools to perform an analysis of the standard market structures. In the following section, we study the relation between conjectures and market power as measured by the Lerner Index. In the subsequent section, we study the link between conjectures and market concentration as measured by the Herfindahl Index. The conclusive section provides a synthesis.

\section{THE CONJECTURAL DUOPOLY MODEL}

We consider a two-player game. The players are firms, labeled 1 and 2, producing a homogeneous good. Their strategies are the output quantities $x_{1}$ and $x_{2}$ they decide to produce, chosen in the strategy sets $\sigma_{1}$ and $\sigma_{2}$, respectively. In our model, $\sigma_{i}=\mathbb{R}_{+}$, with $i=1$, 2. Firm $i$ 's cost function is given by $C_{i}\left(x_{i}\right)=c x_{i}$, with $c \geq 0$. The aggregate output is $X \equiv x_{1}+x_{2}$. Firms face a decreasing linear inverse demand function, defined by $p(X)=a-b X$, with $a>c$ and $b>0$. Firm $i$ 's payoff is given by its profit function $\pi_{i}$ :

$$
\pi_{i}\left(x_{i}, x_{j}\right)=p(X) x_{i}-c x_{i}, \quad i, j=1,2 \text { and } i \neq j .
$$


The specificity of our approach consists in introducing an additional assumption on players' beliefs, reflecting a specific way to envisage strategic interactions.

\section{The Definition of a Conjectural Variation}

In standard two-firm strategic environments, the firm's strategy depends on the decision of its rival. This interaction is captured by the existence of a best-response function for each of the two firms. In some cases, as in the Stackelberg duopoly, one of the two firms-the leader-is aware of having an impact on the rival's strategy when making its decision. The leader uses the best-response function of the other firm - the follower - to compute its optimal strategy. In this framework, the leader has no best-response function: There is an asymmetry in the strategic positions of the two firms.

In the strategic environment as we model it, unlike the Stackelberg framework, both firms believe that their own strategy has a direct influence on the choice made by the rival. Firm $i$ 's beliefs are modeled by an individual function indicating how firm $j$ behaves in reaction to the strategy $x_{i}$ (see notably, Bresnahan 1981, Dixit 1986, and Perloff, Karp, and Golan 2007). This function can be presented as firm $i$ 's conjecture (whether correct or not) about firm $j$ 's bestresponse function. In our linear framework, it is convenient to assume that this function is of the form

$$
\psi_{j}\left(x_{i}\right)=\tilde{x}_{j}+v_{i}\left(x_{i}-\tilde{x}_{i}\right)
$$

where $\left(\tilde{x}_{1}, \tilde{x}_{2}\right)$ is a benchmark profile. ${ }^{4}$ This means that when firm $i$ decides to deviate from $\tilde{x}_{i}$, it expects an increase in firm $j$ 's production by $v_{i}$ for each additional unit it produces. However, when both firms act according to the benchmark profile, none of them expects any reaction of the rival. ${ }^{5}$ The parameters $v_{1}$ and $\nu_{2}$ are the slopes of the conjectured functions $\psi_{1}$ and $\psi_{2}$, respectively. Mathematically, $v_{i}=d \psi_{j}\left(x_{i}\right) / d x_{i}$. These parameters are called conjectural variations. They are taken into account when firms decide on the optimal quantity to produce and derive the conjectural best-response functions, by contrast with the conjectured functions. ${ }^{6}$ As a result of these assumptions, firm $i$ 's profit function in equation (1) may be rewritten as

$$
\pi_{i}\left(x_{i}, \psi_{j}\left(x_{i}\right)\right)=p\left(x_{i}+\psi_{j}\left(x_{i}\right)\right) x_{i}-c x_{i}
$$

We use the profit above in the remainder of this article to capture the different market structures (including perfect competition, monopoly, and the standard duopolies), whose individual and aggregate outcomes explicitly depend on conjectures.

\section{The Conjectural Duopoly Equilibrium}

Each firm maximizes the profit function in equation (3), relying on the conjectures expressed in equation (2). The first-order conditions lead to

$$
\pi_{i}{ }^{\prime}\left(x_{i}, \psi_{j}\left(x_{i}\right)\right)=\underbrace{a-b X}_{p(X)+p^{\prime}(X)} \underbrace{-b}\left(1+v_{i}\right) x_{i}-c=0 .
$$

From these conditions, which are trivially necessary and sufficient given that both the inverse demand and the conjectured best-response functions are linear, we derive the following conjectural 
best-response functions:

$$
x_{i}=\frac{a-c}{b\left(2+v_{i}\right)}-\frac{1}{2+v_{i}} x_{j}
$$

They are similar to the standard best-response functions of a game, except that they explicitly embody conjectures. Firm i's optimal strategy depends on firm $j$ 's strategy, but also on its own conjectural variation $v_{i}$. The strategy is then modified in accordance with conjectures. Equivalently, we can express the strategy as a share of the perfect competition output $X^{*}$ to exhibit the market power of firms; that is, their ability to rationally restrict output below the competitive outcome and set a market price above the marginal cost. In our linear economy, $X^{*}=(a-c) / b$ (see next section for more details). The conjectural best-response functions in (5) can be rewritten as

$$
\frac{x_{i}}{X^{*}}=\frac{1}{2+v_{i}}-\frac{1}{2+v_{i}} \frac{x_{j}}{X^{*}}
$$

The conjectural best-response functions so-obtained are mandatory to compute the conjectural duopoly equilibrium $\tilde{x}$.

Definition (Conjectural duopoly equilibrium). A conjectural duopoly equilibrium consists of a strategy profile $\tilde{x}=\left(\tilde{x}_{1}, \tilde{x}_{2}\right)$ and a pair of conjectures $v=\left(v_{1}, v_{2}\right)$ such that for all $x_{i} \in \sigma_{i}$ : $\pi_{i}\left(\tilde{x}_{i}, \psi_{j}\left(\tilde{x}_{i}\right)\right) \geq \pi_{i}\left(x_{i}, \psi_{j}\left(x_{i}\right)\right)$ with $\psi_{j}\left(x_{i}\right)=\tilde{x}_{j}+v_{i}\left(x_{i}-\tilde{x}_{i}\right), i=1,2$ and $i \neq j$.

The conjectural equilibrium is such that no firm has an incentive to deviate unilaterally from $\tilde{x}$ given the conjectural variations $v_{1}$ and $v_{2}$ formed by the two players. In other words, $\tilde{x}_{i}$ maximizes firm $i$ 's profit when it conjectures a reaction function for firm $j$ of the form $\psi_{j}\left(x_{i}\right)=$ $\tilde{x}_{j}+v_{i}\left(x_{i}-\tilde{x}_{i}\right)$ and provided the strategy $\tilde{x}_{j}$ of the rival remains unchanged. Stated differently, the strategy profile $\tilde{x}=\left(\tilde{x}_{1}, \tilde{x}_{2}\right)$ is attained when the conjectural best-response functions are both mutually compatible. In our linear framework, the conjectural equilibrium is given by

$$
\left(\frac{\tilde{x}_{1}}{X^{*}}, \frac{\tilde{x}_{2}}{X^{*}}\right)=\left(\frac{1+v_{2}}{\left(2+v_{1}\right)\left(2+v_{2}\right)-1}, \frac{1+v_{1}}{\left(2+v_{1}\right)\left(2+v_{2}\right)-1}\right)
$$

Equation (7) is useful to reproduce without further derivation the standard market structures and specific outcomes covered by the economic textbooks (as well as many others). Each of them appears as a special case of conjectural equilibrium for an accurate choice of the conjectures $v_{1}$ and $v_{2}$. The corresponding equilibrium profit for firm $i$ is

$$
\tilde{\pi}_{i}\left(v_{i}, v_{j}\right)=\frac{(a-c)^{2}}{b} \frac{\left(1+v_{i}\right)\left(1+v_{j}\right)^{2}}{\left[\left(2+v_{i}\right)\left(2+v_{j}\right)-1\right]^{2}}
$$

The profit is decreasing with the firm's conjecture and is increasing with its rival's. As shown infra, the conjecture value is closely related to the degree of cooperation adopted by the firm. While it is detrimental to one firm to be more cooperative, it proves beneficial to the other.

\section{Market Structures and Conjectural Variations}

In this section, we review the pairs of conjectures parameterizing the standard market structures, including perfect competition, the Cournot duopoly, the Stackelberg duopoly, and collusion. We 
determine the outcomes graphically and analytically, using only the conjectural best-response functions given by equation (6).

\section{The Competitive Market Outcome}

The competitive outcome $X^{*}$ is obtained when price is equal to marginal cost, that is, $p\left(X^{*}\right)=c$, and computed from the definition of the inverse demand function:

$$
X^{*}=\frac{a-c}{b}
$$

This aggregate output may be obtained from equation (4) by imposing $v_{1}=v_{2}=-1$. The conjectures mean that each firm expects the rival to compensate exactly any expansion or reduction in its own supply, leaving the equilibrium price unchanged. In such a case, firms have the perception that they cannot manipulate the market price strategically and can be considered as price takers. The conjectural best-response functions in (6) both reduce to

$$
\frac{x_{i}}{X^{*}}=1-\frac{x_{j}}{X^{*}} \text { or } \frac{x_{i}}{X^{*}}+\frac{x_{j}}{X^{*}}=1,
$$

meaning that the two firms share the global competitive output $X^{*}$. The way the output is split up across firms is indeterminate. This property is unrelated to conjectural variations but stems from the constant marginal cost. In the continuum of feasible competitive distributions, corresponding to the line segment $\overline{P_{1} P_{2}}$ in figure 1 , the equilibria can be indexed by the parameter $\alpha \in[0,1]$, standing for the share devoted to firm 1 . The set of competitive equilibria (expressed in terms

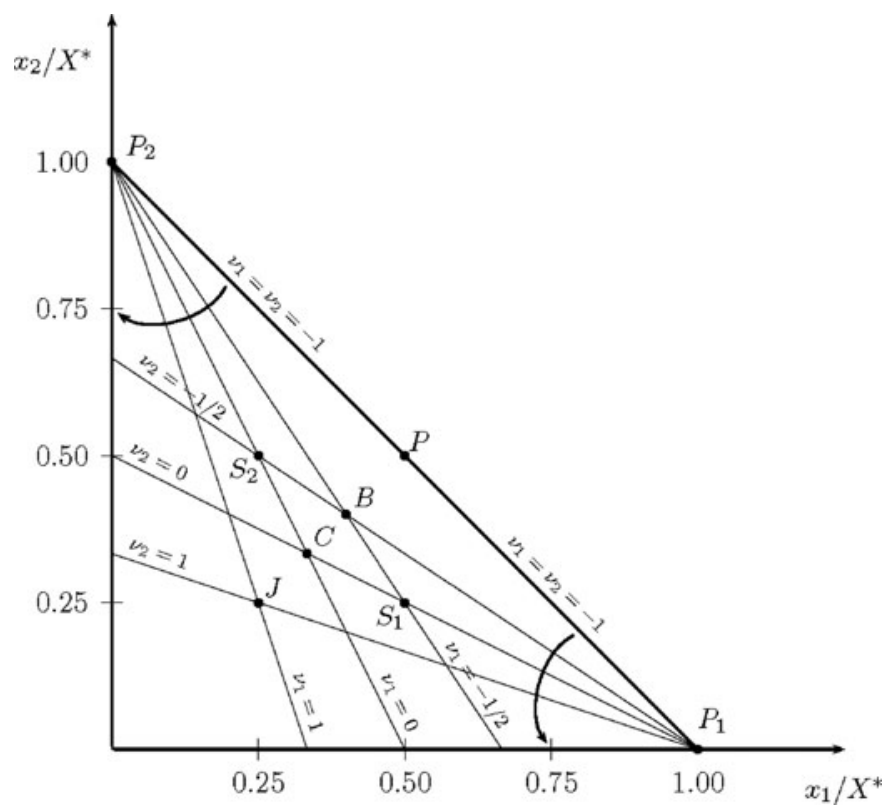

FIGURE 1 Conjectural variations and the standard market structures. 
of market shares) is given by $\left(\tilde{x}_{1} / X^{*}, \tilde{x}_{2} / X^{*}\right)=(\alpha, 1-\alpha)$. In textbooks, symmetry is usually assumed. The competitive equilibrium is then presented as the specific case $P=(1 / 2,1 / 2)$, corresponding to $\alpha=1 / 2$.

Notice that the competitive outcome may also be obtained if one of the two firms - say $i$-forms the conjecture $v_{i}=-1$ while the other has a conjecture $v_{j}>-1$. In this socially efficient context, firm $i$ behaves as a price taker (in the sense indicated above) and produces the whole output (the best strategy for firm $j$ is $\tilde{x}_{j}=0$ ), charging a price just equal to marginal cost although it is the unique producer. These two polar cases correspond to the points $P_{1}$ and $P_{2}$ in figure 1 .

\section{The Cournot Market Outcome}

In the Cournot-Nash duopoly, a firm does not expect any reaction from the other to a change in its own supply (as if it considered the strategy of the rival as inelastic when determining its optimal choice). The Cournot conjectures are then such that $v_{i}=0$ for all $i$. From equation (6), the corresponding best-response functions are then given by

$$
\frac{x_{i}}{X^{*}}=\frac{1}{2}-\frac{1}{2} \frac{x_{j}}{X^{*}}, \quad i=1,2 .
$$

They logically coincide with the best-response functions of the nonconjectural Cournot duopoly and yield the standard equilibrium strategies $\left(\tilde{x}_{1} / X^{*}, \tilde{x}_{2} / X^{*}\right)=(1 / 3,1 / 3)$, easily deduced from equation (7).

\section{The Stackelberg Market Outcomes}

The nonconjectural Stackelberg setup is sequential. The leader decides first the individual quantity to produce. The follower observes this quantity and determines its production accordingly. The leader knows the follower's best-response function and uses it to compute its optimal strategy. Under the assumption of linear demand and costs, the leader's strategy is twice as large as the follower's strategy: Firms globally produce three-quarters of the competitive aggregate output. In our conjectural framework, they always choose their strategies simultaneously. The pair of conjectures $\left(v_{i}, v_{j}\right)=(-1 / 2,0)$ is able to reproduce the behavior of the two firms when firm $i$ behaves as a leader. According to the conjectures, the latter considers that firm $j$ absorbs half the fraction of any increase in its own supply (corresponding to the slope of the best-response function). The conjecture $v_{j}=0$ of firm $j$ means that it believes it has no influence on firm $i$ 's strategy. The corresponding conjectural best-response functions are (respectively for the leader and the follower)

$$
\begin{aligned}
& \frac{x_{i}}{X^{*}}=\frac{2}{3}-\frac{2}{3} \frac{x_{j}}{X^{*}}, \\
& \frac{x_{j}}{X^{*}}=\frac{1}{2}-\frac{1}{2} \frac{x_{i}}{X^{*}} .
\end{aligned}
$$

Depending on the relative positions of leader and follower adopted by firms 1 and 2, the Stackelberg outcome can be either $\left(\tilde{x}_{1} / X^{*}, \tilde{x}_{2} / X^{*}\right)=(1 / 2,1 / 4)$ or $\left(\tilde{x}_{1} / X^{*}, \tilde{x}_{2} / X^{*}\right)=(1 / 4,1 / 2)$, corresponding respectively to $S_{1}$ and $S_{2}$ in figure 1 . 


\section{The "Stackelberg Warfare"}

An unusual equilibrium configuration occurs when each firm considers itself as a leader. In our framework, they share the same conjecture $v_{1}=v_{2}=-1 / 2$ and an identical conjectural best-response function, expressed in equation (9a) and corresponding to the optimal choice of a Stackelberg leader. The equilibrium $B$ is graphically determined in figure 1 by the intersection of the two conjectural best responses. This double-leader situation, sometimes referred to as the Stackelberg warfare, was originally envisaged by Bowley (1924) and Stackelberg (1934/2011). This outcome cannot be calculated using the standard Stackelberg duopoly resolution because leaders do not have any best-response function. In textbooks, a solution to the problem is usually obtained through the assumption that each firm considers its rival as a follower (e.g., Picard 2007), resulting in a rationally inconsistent outcome, with two leaders assuming the other firm acts as follower. In our framework, by contrast, firms form peculiar conjectures but do not make any mistake about their rival's behavior. The resulting outcome is $\left(\tilde{x}_{1} / X^{*}, \tilde{x}_{2} / X^{*}\right)=(2 / 5,2 / 5)$. Strategies are lower than those displayed in Picard (2007), where both firms play the leader's strategy presented in the above section.

\section{The Collusive Market Outcome}

Under symmetric collusion, firms maximize the joint profit and equally share the corresponding production. In terms of conjectures, each of them internalizes the impact of their strategy on both its partner's profit and the aggregate output. A one-unit rise in its individual production is assumed to imply an additional unit in the production of the partner, that is, $v_{1}=v_{2}=1$. The conjectural best-response function of firm $i$ is then

$$
\frac{x_{i}}{X^{*}}=\frac{1}{3}-\frac{1}{3} \frac{x_{j}}{X^{*}}
$$

leading to the market outcome $\left(\tilde{x}_{1} / X^{*}, \tilde{x}_{2} / X^{*}\right)=(1 / 4,1 / 4)$.

The aggregate output corresponds to the production of a monopoly. It could be shared differently between the firms: From equation (7), any pair of conjectures satisfying $v_{1} v_{2}=1$ leads to the minimal aggregate output of $1 / 2$. The higher the firm's conjecture is, the lower its production in the collusive sharing. The corner cases $(1 / 2,0)$ and $(0,1 / 2)$ correspond to effective monopoly situations.

\section{Conjectural Equilibrium: Graphical Representation}

The previous conjectural equilibria are represented graphically in figure 1 . They all emerge from the intersection of the individual conjectural best-response functions. Points $C$ and $J$ correspond respectively to the Cournot and the symmetric collusive outcomes. Points $S_{1}$ and $S_{2}$ stand for the two Stackelberg equilibria, respectively with firm 1 and firm 2 as leaders, while point $B$ is the Bowley equilibrium. Among the many possible competitive equilibria, we retain the symmetric one (point $P$ ).

In figure 1 , an individual conjectural variation may range from -1 to $+\infty .^{7}$ When the value of the conjectures rises, the best-response functions rotate down: Firms adopt a more cooperative 
behavior, restricting the individual outputs, then the aggregate output, which benefits to both of them (as long as market shares remain unchanged).

As indicated in the introduction, conjectural variations can also be used to investigate aggregate measures of market power, such as the Lerner and the Herfindahl indexes. We represent these measures graphically in our framework as an alternative approach to the conjectural best-response functions studied in the current section. We show that any pair of conjectures uniquely determines not only a couple of conjectural best-response functions but also a couple of Lerner and Herfindahl indexes, and fully characterizes the equilibrium market structure and outcome.

\section{MARKET POWER IN DUOPOLY MODELS: THE ISO-LERNER LINE}

One of the most widely used indexes in the literature is probably the Lerner Index, which offers a synthetic way to measure market power. It is computed as the difference between market price and marginal cost, expressed as a percentage of the price. It is bounded below by zero, breaks even in a competitive equilibrium, and increases as firms reduce aggregate output.

\section{Measuring the Market Power Using Conjectures}

Perfect competition and monopoly constitute the polar cases for studying both market power and the form of competition. In a nonconjectural approach, they cannot be compared directly because they assume irreconcilable hypotheses: Firms are price takers in perfect competition while they are price makers otherwise. ${ }^{8}$ In our framework, comparisons are allowed by a continuous change in a competition index defined by Perloff and colleagues (2007), denoted by $\lambda$. It evolves according to values of the conjectural variations.

The index appears in the equilibrium aggregate output, obtained from equation (7) after some algebra:

$$
\tilde{X} \equiv \tilde{x}_{1}+\tilde{x}_{2}=\frac{1}{1+\lambda} \frac{a-c}{b} \quad \text { with } \quad \lambda=\left\{\begin{array}{l}
0 \text { for } v_{1}=-1 \text { or } v_{2}=-1 \\
1-\frac{1-v_{1} \nu_{2}}{2+v_{1}+v_{2}} \text { otherwise }
\end{array}\right.
$$

To make sure that the price lies between the marginal cost and the monopoly price, the $\lambda$ index must range from 0 to 1 . The conjectural variations, bounded below by -1 to insure nonnegative profits, are then also rationally constrained by the inequation $1-v_{1} v_{2} \geq 0$. The higher $\lambda$, the lower the equilibrium aggregate output and the higher the equilibrium price. Market power of firms is parameterized by the conjectural variations and may be defined with respect to the Lerner Index $L$ by

$$
L(\lambda) \equiv \frac{p-c}{p}=\frac{\lambda}{|\varepsilon|}
$$

where $\varepsilon \equiv-(a-b X) / b X$ is the price elasticity of demand, measuring the responsiveness of global demand to a change in the market price. ${ }^{9}$ The individual elasticities faced by firms are not relevant in our framework with identical prices and marginal costs. 


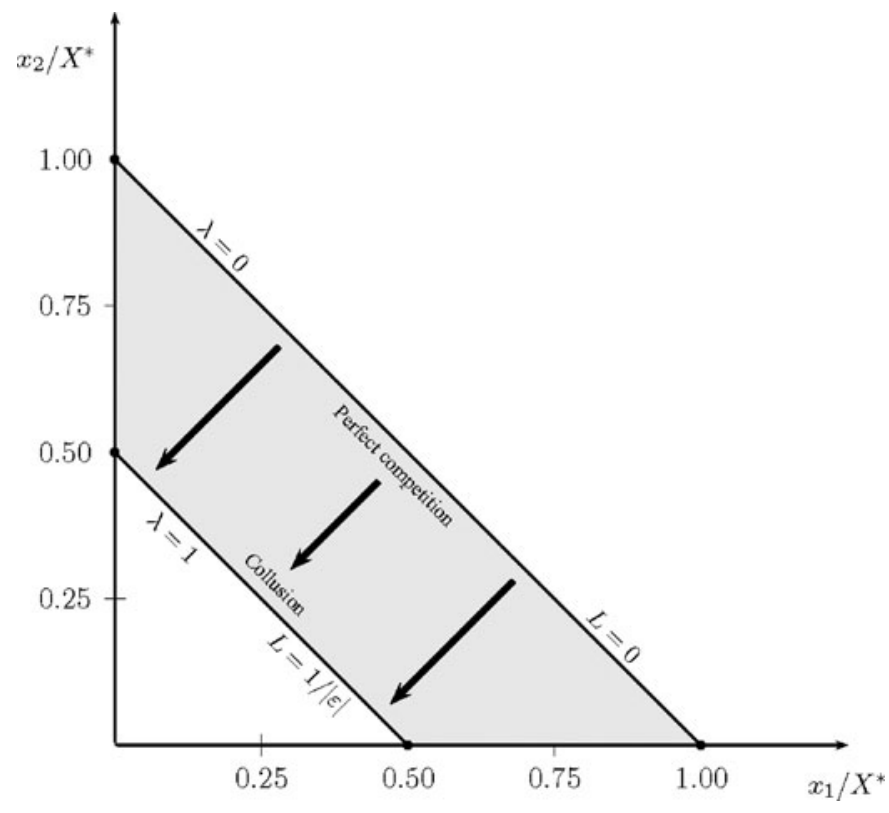

FIGURE 2 The Lerner line (for $\lambda=0, \lambda=1$, and the intermediate cases).

\section{The Iso-Lerner Line}

For any given value of $\lambda \in[0,1]$, the aggregate output $X$ is unique and so is the price elasticity of demand $\varepsilon$. As a consequence, there is a one-to-one relationship between $\lambda$ and the Lerner Index. As $\lambda$ increases from zero, the aggregate output goes down while the equilibrium price goes up: The price elasticity of demand drops (in absolute value), and the Lerner Index finally increases.

Using (10), one can determine a linear relationship between the two shares $x_{1} / X^{*}$ and $x_{2} / X^{*}$ :

$$
\frac{x_{2}}{X^{*}}=\frac{1}{1+\lambda}-\frac{x_{1}}{X^{*}}
$$

The graph of this functional relationship is called an iso-Lerner line: All pairs $\left(x_{1} / X^{*}, x_{2} / X^{*}\right)$ along the line are associated with the same $\lambda$, thus the same Lerner Index.

The entire set of iso-Lerner lines is sketched in figure 2 for the different values of $\lambda$, in the same space as in figure 1 . They are decreasing and shift down as $\lambda$ increases.

\section{Market Structures and Iso-Lerner Lines}

As we can see from equation (12), the slope of an iso-Lerner line is always equal to -1 : An increase in $\lambda$ only affects the intercept, whose level corresponds to aggregate output (expressed in proportion of the competitive output). With a Lerner Index and a $\lambda$ equal to zero, the farthest line from the origin corresponds to perfect competition. This iso-Lerner line pictures the continuum of feasible aggregate output divisions between the two firms. The coordinates $(\alpha, 1-\alpha)$ of a 
point lying on the line indicate the share of the competitive aggregate output devoted to firm 1 and 2 , respectively.

As $\lambda$ and $L$ increase from zero, aggregate output decreases, and the market power of firms becomes stronger, causing the iso-Lerner line to shift down toward the origin. Collusion is reached for the maximum value $\lambda=1$ and a Lerner Index of $1 /|\varepsilon|$. The intermediate cases $\lambda \in(0,1)$ capture oligopoly market structures, including the Cournot and the Stackelberg outcomes.

The iso-Lerner lines visually help to rank the different equilibria in accordance with the degree of market power, identical across firms (they face the same price and have the same marginal cost). For instance, in figure 1, it can be observed graphically that the sequential Stackelberg duopoly, with a Lerner Index of $L=1 /|3 \varepsilon|$ and an intercept of $2 / 3$, lowers market power compared to the simultaneous Cournot game $(L=1 /|2 \varepsilon|$ and an intercept of $1 / 3)$.

\section{MARKET CONCENTRATION IN DUOPOLY MODELS: THE ISO-HERFINDAHL LINE}

The Herfindahl Index is sometimes used as an alternative to the Lerner Index for measuring market power. It is built on the asymmetry in the market shares of firms. A market with a deep asymmetry is considered as being less competitive because it implies a dominant position for one of the two firms, resulting in an excess of market power. In our framework, under the assumption of identical marginal cost for all firms, the Herfindahl Index is not suited to measure market power. However, it remains accurate for studying market concentration and constitutes a good complement to the Lerner Index to describe the market structure. More precisely, the combination of the two indexes is sufficient to fully characterize the equilibrium outcome.

\section{Measuring Market Concentration Using Conjectural Variations}

The Herfindahl Index, denoted by $H$, is defined as the sum of the squares of the market shares of firms, that is

$$
H\left(x_{1}, x_{2}\right) \equiv \sum_{i=1}^{2}\left(\frac{x_{i}}{X}\right)^{2}=\left(\frac{x_{1}}{x_{1}+x_{2}}\right)^{2}+\left(\frac{x_{2}}{x_{1}+x_{2}}\right)^{2}
$$

Symmetric equilibria display the same Herfindahl Index, equal to $1 / 2$. The index rises as soon as the asymmetry in market shares become stronger. For example, the two Stackelberg equilibria are associated with a Herfindahl Index $H=5 / 9$.

\section{The Iso-Herfindahl Line}

Notice that the functional relationship in (13) is homothetic: For any $k>0, H\left(k x_{1}, k x_{2}\right)=$ $H\left(x_{1}, x_{2}\right)$. As a consequence, any pair of outcomes $x$ and $x^{\prime}$, with $x_{1}^{\prime}=k x_{1}$ and $x_{2}^{\prime}=k x_{2}$, have the same Herfindahl Index. Denote by $\eta$ the ratio

$$
\eta \equiv \frac{x_{2}}{x_{1}}=\frac{x_{2} / X}{x_{1} / X}
$$




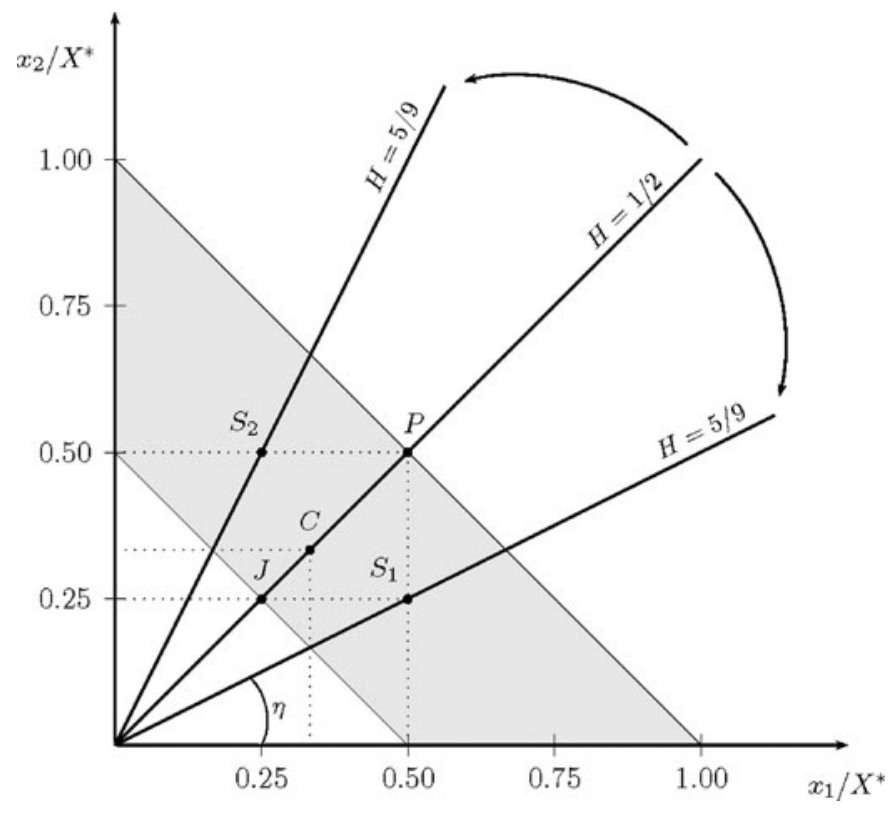

FIGURE 3 The Herfindahl line (for $\eta=1 / 2, \eta=1$, and $\eta=2$ ).

This coefficient is included between 0 (when firm 1 produces the whole aggregate output) and $+\infty$ (when firm 2 is the unique producer). From the homothetic property of $H$, it can be easily deduced that the different outcomes characterized by the same coefficient $\eta$ share a unique Herfindahl Index. In the plane $\left(x_{1} / X^{*}, x_{2} / X^{*}\right)$, these outcomes form a half-ray whose equation is given by

$$
\frac{x_{2}}{X^{*}}=\eta \frac{x_{1}}{X^{*}}
$$

obtained after rearranging equation (14). These half-rays are called iso-Herfindahl lines. They are upward sloping because $\eta \geq 0$ and pass through the origin. ${ }^{10}$ In figure 3 , we pictured the iso-Herfindahl lines for the two conjectural Stackelberg equilibria $S_{1}$ and $S_{2}$. The Herfindahl Index is identical in both cases.

By contrast with the Lerner Index, the Herfindahl Index is not associated with a unique line but two, except for $H=1 / 2$. In this latter case (symmetric equilibria), the iso-Herfindahl line merges with the first bisector (see points $J, C$, and $P$ ). Graphically, the same rotations of the line from $\eta=1$ in one direction or the other have an identical impact on the Herfindahl Index but differ in the dominant firm that emerges (firm 1 on the right, firm 2 on the left).

\section{Market Structures and Iso-Herfindahl Lines}

The iso-Herfindahl line $H=0$ is coincident with the $x$-axis. It gathers together the different market structures whose aggregate outcome is uniquely produced by firm 1, including the standard monopoly structure $(1 / 2,0)$ ). For $\eta<1$ (resp. $\eta>1$ ), firm 1 (resp. 2) enjoys a dominant position 
on the market. As $\eta$ increases from 0 , firm 1 faces a diminishing market share. When $\eta$ tends to infinity, the iso-Herfindahl line is coincident with the $y$-axis, and the aggregate outcome is entirely produced by firm 2 . In any case, along a given iso-Herfindahl line, different degrees of competition are associated with a unique aggregate output division.

To the contrary, any degree of market power is associated with a multiplicity (or even a continuum) of aggregate output divisions between the two firms. For instance, the competitive market outcome is compatible with the whole set of iso-Herfindahl lines, each of them crossing the iso-Lerner line $L=0$. As said before, the aggregate output repartition is indeterminate: The higher $\eta$, the higher the market share devoted to firm 2 . The standard symmetric perfect competition $P$ is only a particular case, obtained for $\eta=1$. Collusive outcomes exhibit a similar pattern: They share the same iso-Lerner line but lie on different iso-Herfindahl lines.

In a sense, the iso-Lerner line is more informative about market power in our model than the iso-Herfindahl line (the latter being interesting to get information about the degree of market concentration). The contrast between the multiplicity of $H$ and the uniqueness of $L$ to evaluate market power does not come from the conjectural variations but rather from the assumption that firms face the same market price and have identical marginal costs.

At last, it is worth noting that the Lerner and the Herfindahl indexes may have opposite conclusions; for instance, consider both the Stackelberg and the Cournot outcomes. The Herfindahl Index is higher for the former duopoly structure $(H=5 / 9)$, indicating a higher degree of concentration: The leader produces twice the follower's output. However, the Cournot duopoly displays a higher Lerner Index $(L=1 /|2 \varepsilon|$ for Cournot and $L=1 /|3 \varepsilon|$ for Stackelberg), meaning that market distortions are stronger: Firms exert more market power when they behave in a Cournotian way.

\section{GRAPHICAL DETERMINATION OF MARKET OUTCOMES USING THE ISO-LERNER AND THE ISO-HERFINDAHL LINES}

There is a direct connection between the Lerner and Herfindahl indexes on one hand and the conjectural variations on the other hand: Choosing a pair $\left(v_{1}, v_{2}\right)$ is tantamount to choosing a pair $(L, H)$. This one-to-one correspondence can be easily observed in figure 4 .

All the conjectural equilibria belong to the gray trapezoid delimited by the competitive and the collusive Lerner lines. Any market structure can emerge graphically either by combining the conjectural best responses of the two firms or the iso-Lerner and the iso-Herfindahl lines. In figure 4, the Stackelberg outcome $S_{1}$ is pictured using the two alternative methods. Firm 1 acts as the leader, with a best-response function characterized by $v_{1}=-1 / 2$, while firm 2 endorses the follower's conjecture $v_{2}=0$. Therefore, the equilibrium $\left(\tilde{x}_{1} / X^{*}, \tilde{x}_{2} / X^{*}\right)=(1 / 2,1 / 4)$ appears as the intersection of both

- The conjectural best-response functions:

$$
\begin{aligned}
& \frac{x_{1}}{X^{*}}=\frac{2}{3}-\frac{2}{3} \frac{x_{2}}{X^{*}} \text { for firm } 1 \\
& \frac{x_{2}}{X^{*}}=\frac{1}{2}-\frac{1}{2} \frac{x_{1}}{X^{*}} \text { for firm2. }
\end{aligned}
$$

- The iso-Lerner and the iso-Herfindahl lines, respectively $L=1 /|3 \varepsilon|$ and $H=5 / 9$. 


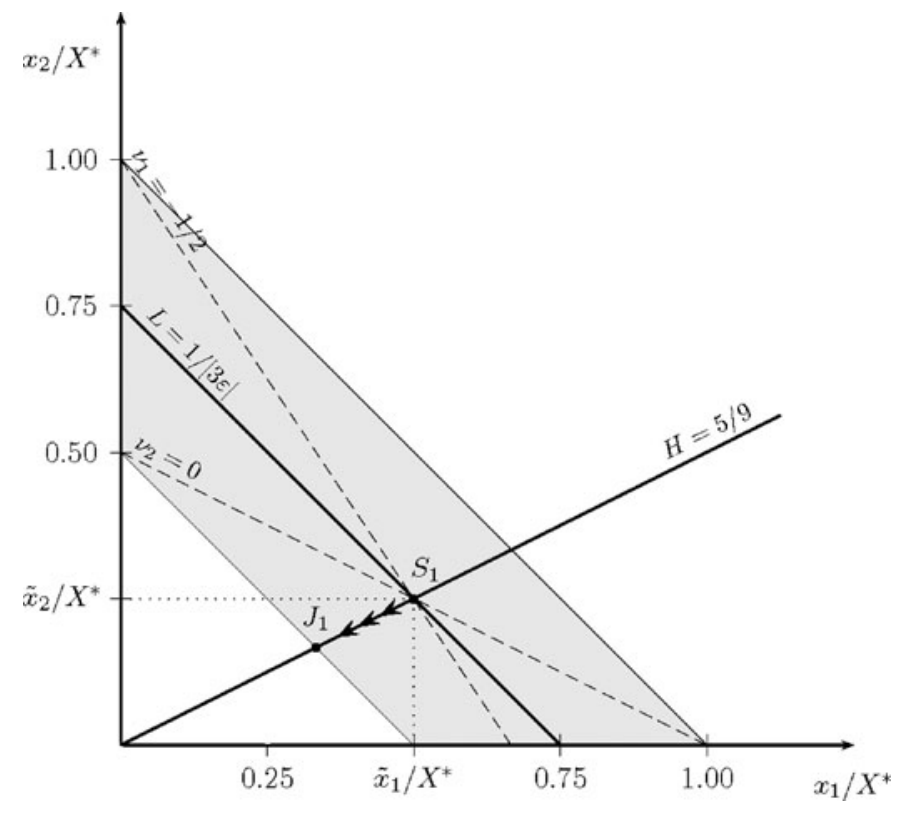

FIGURE 4 The conjectural Stackelberg equilibrium (firm 1 as leader).

While the economic literature covers various degrees of market power, it usually restricts the analysis to symmetric equilibria (with the mere exception of the Stackelberg duopoly). The conjectural approach exposed in this article is useful to enlarge the analysis to asymmetric structures and market concentration, and makes worthwhile studying the Herfindahl Index in models with linear demand and identically constant marginal costs. Combining (7) and (14) yields

$$
\eta=\frac{1+v_{2}}{1+v_{1}}
$$

As said before, the value $\eta=1$ corresponds to a market outcome in which firms have an identical market share (symmetric equilibrium). It is obtained for $v_{1}=v_{2}$. The Herfindahl Index is minimal, equal to $H=1 / 2$. Market concentration crucially depends on the asymmetry between $v_{1}$ and $v_{2}$ : The stronger the asymmetry, the more concentrated the market will be. When $v_{1}<v_{2}$ (resp. $v_{1}>v_{2}$ ), that is $\eta<1$ (resp. $\eta>1$ ), firm 1 (resp. 2), expecting a weak reaction of firm 2 (resp. 1) to an additional amount of output, produces a higher part of the aggregate output. Trivially, the market structures characterized by the pairs of conjectural variations $\left(v_{1}, v_{2}\right)$ and $\left(v_{2}, v_{1}\right)$ display an identical degree of concentration. The corresponding iso-Herfindahl lines are symmetrical about the first bisector.

The coefficient $\lambda$ is directly linked to the competitive behavior of firms, which is in turn summarized by the conjectural variations. According to equation (10), the higher $v_{1}$ and $v_{2}$, the lower $\lambda$ and the Lerner Index will be. From the conjectural Stackelberg equilibrium $S_{1}$ of figure 4, the two firms may increase market power by decreasing the individual outputs proportionally such 
that they preserve the relative market share $\eta$. The Lerner Index then increases from $L=1 /|3 \varepsilon|$ to $L=1 /|\varepsilon|$, while the Herfindahl Index remains unchanged. The new conjectural equilibrium so obtained is $J_{l}$, for which the aggregate profit is maximal. More generally, any asymmetric collusive equilibrium can be analyzed as the endpoint of a converging process toward a more collusive market structure, decreasing the aggregate output and holding constant market shares.

This process clearly increases the individual profits that can be expressed analytically using both the Lerner Index and the relative market share $\eta$ :

$$
\begin{aligned}
& \tilde{\pi}_{1}(\lambda, \eta)=\frac{\lambda}{(1+\lambda)^{2}(1+\eta)} \frac{(a-c)^{2}}{b} \\
& \tilde{\pi}_{2}(\lambda, \eta)=\frac{\lambda}{(1+\lambda)^{2}\left(1+\eta^{-1}\right)} \frac{(a-c)^{2}}{b} .
\end{aligned}
$$

Profits are increasing with the Lerner Index and rise with the aggregate market power. Straightforwardly, the relative market share affects differently the profits, decreasing firm 1's profit and increasing firm 2's profit.

As said before, the Cournot duopoly displays a higher Lerner Index but a lower Herfindahl Index as the Stackelberg duopoly, indicating a lower degree of market power but a higher degree of concentration. It can be deduced from equation (8) that the overall effect on the leader's profit is positive because $v_{1}$ is lower in the Stackelberg duopoly while $v_{2}$ remains unchanged. The decrease in the market price is then more than compensated by the increase in the leader's market share.

\section{SYNTHESIS AND CONCLUSION}

We now summarize in table 1 the duopoly outcomes presented in the previous sections. We give the pair of conjectures associated with each conjectural equilibrium and the corresponding Lerner and Herfindahl indexes.

In our framework, market power does not depend only on fundamentals (technology and demand) but also on the conjectures formed by the players. The purpose of this approach is not to replace the standard presentations of market structures, but rather to complement them. Hence, it gives some additional insights for students to understand the diversity of competition. For instance, by founding the competitive behavior on individual beliefs about the response of other firms, rather than on strategic isolation or on the number of firms, it provides an alternative viewpoint to the concept of price-taking behavior. Conjectural variations as a synthesis of chapters on oligopoly have already been used, for example, in Varian (1993) or Estrin and colleagues (2008). However, these textbooks usually confine themselves to a short reference to the topic. In this article, we proposed an exhaustive investigation with additional elements, including a unified analytical treatment of the different market structures and an original graphical representation of firm behaviors, market outcomes, and aggregate measures of market power and concentration.

The study of market structures with conjectural variations can be extended even further to encompass welfare analysis. With respect to the aims of IO as stated in the introduction, conjectural variations provide a convenient framework to investigate global welfare: They precisely refer to an implicit degree of collusion between firms, the collusive behavior rising with the values of the conjectures. 
TABLE 1

The Conjectural Synthesis

\begin{tabular}{lcccc}
\hline Framework & $v_{1}$ & $v_{2}$ & $\left(\tilde{x}_{1} / X^{*}, \tilde{x}_{2} / X^{*}\right)$ & $L$ \\
\hline Symmetric collusion $(J)$ & 1 & 1 & $(1 / 4,1 / 4)$ & $1 /|\varepsilon|$ \\
Cournot duopoly $(C)$ & 0 & 0 & $(1 / 3,1 / 3)$ & $1 / 2$ \\
Stackelberg duopoly 1 $\left(S_{1}\right)$ & $-1 / 2$ & 0 & $(1 / 2,1 / 4)$ & $1 / 2 \varepsilon \mid$ \\
Stackelberg duopoly 2 $\left(S_{2}\right)$ & 0 & $-1 / 2$ & $(1 / 4,1 / 2)$ & $1 /|3 \varepsilon|$ \\
Bowley duopoly $(B)$ & $-1 / 2$ & $-1 / 2$ & $(2 / 5,2 / 5)$ & $1 / 2 / 4 \varepsilon \mid$ \\
Perfect competition $(P)$ & -1 & -1 & $(1 / 2,1 / 2)$ & $1 / 2$ \\
\end{tabular}

\section{NOTES}

1. This approach has been adopted, for instance, by Varian (1993) or Serrano and Feldman (2013), among others.

2. Other pedagogical presentations of the Cournot oligopoly have been made by Sarkar, Gupta, and Pal (1998) and Dufwenberg (2001), but they insist on simpler presentations of the model.

3. A static conjectural variation equilibrium can be seen as the reduced form of a repeated game (Cabral 1995), which is not here explicitly modeled and is out of the scope of the article.

4. This benchmark profile will be defined in the next section as the equilibrium of the game. However, for pedagogical reasons, it seems more convenient to specify it later, when the notion of conjectural equilibrium is introduced.

5. In the literature, the conjectured best-response functions are not specified. Only the $v$ s, defined as the derivatives of the $\psi \mathrm{s}$ at the benchmark profile, are compulsory for the resolution of the model. However, for sake of clarity, it is appropriate to consider a specific form for the best-response function. We are indebted to an anonymous referee for making this proposal.

6. The relevant literature makes the distinction between the conjectured and the true best-response functions. The latter is usually called the conjectural best-response function and is determined as the solution of the maximization program of the firm. The conjectured best-response function can be envisaged as the reduced form of an unspecified dynamic game. For more details, see Cabral (1995) or Figuières et al. (2004).

7. The reader familiar with conjectural variations can be surprised by the upper bound of the conjectures, usually set to 1 . The upper bound is in fact dictated by the constraint $v_{1} v_{2}=1$, associated with the minimal aggregate output. The standard presentations assuming symmetric conjectures then impose $v_{1}=v_{2}=1$. However, with asymmetric conjectures, any combination satisfying the constraint is feasible; this does not require any upper bound for the individual conjectures.

8. In textbooks, comparisons between the two cases are realized through general comments on price, quantities, and welfare.

9. Vives (2001) proposed an alternative formulation of the relationship between conjectural variations and the Lerner Index for a firm in the symmetric case.

10. For simplicity, the slope of an iso-Herfindahl line is represented in figure 3 as an angle. However, the corresponding angle is equal to $\arctan \eta$.

\section{ACKNOWLEDGEMENTS}

The authors are especially grateful to an anonymous referee whose comments and suggestions deeply improved the content of this paper. Any remaining errors are the authors' sole responsibility. 


\section{REFERENCES}

Bowley, A. L. 1924. The mathematical groundwork of economics. Oxford, UK: Oxford University Press. Bresnahan, T. 1981. Duopoly with consistent conjectures. American Economic Review 71(5): 934-45.

Cabral, L. 1995. Conjectural variations as a reduced form. Economics Letters 49(4): 397-402. 2000. Introduction to industrial organization. Cambridge, MA: MIT Press.

Carlton, D. W., and J. M. Perloff. 1994. Modern industrial organization. 2nd ed. New York: HarperCollins College Publishers.

D'Agata, A. 2010. Geometry of Cournot-Nash equilibrium with application to commons and anticommons. Journal of Economic Education 41: 169-76.

Dixit, A. 1986. Comparative statics for oligopoly. International Economic Review 27(1): 107-22.

Dufwenberg, M. 2001. Teaching Cournot without derivatives. Journal of Economic Education 32: 36-40.

Einat, L., and J. Levin. 2010. Empirical industrial organization: A progress report. Journal of Economic Perspectives 24(2): 145-62.

Estrin, S., D. Laidler, and M. Dietrich. 2008. Microeconomics. 5th ed. Harlow, UK: Pearson Education Ltd.

Figuières, C., A. Jean-Marie, N. Quérou, and M. Tidball. 2004. Theory of conjectural variations. London: World Scientific Publishing.

Fulton, M. 1997. A graphical analysis of the Cournot-Nash and Stackelberg models. Journal of Economic Education 28: 48-57.

McMillan, J. 1986. Game theory in international economics. New York: Harwood Academic Publishers.

Perloff, J. M., L. S. Karp, and A. Golan. 2007. Estimating market power and strategies. New York: Cambridge University Press.

Picard, P. 2007. Eléments de microéconomie. 7th ed. Paris: Montchrestien.

Sarkar, J., B. Gupta, and D. Pal. 1998. A geometric solution of a Cournot oligopoly with nonidentical firms. Journal of Economic Education 29: 118-26.

Serrano, R., and A. M. Feldman. 2013. A short course in intermediate microeconomics with calculus. Cambridge, UK: Cambridge University Press.

Tirole, J. 1988. The theory of industrial organization. Cambridge, MA: MIT Press.

Varian, H. 1993. Intermediate microeconomics. 3rd ed. New York: W. Norton Company.

Vives, X. 2001. Oligopoly pricing: Old ideas and new tools. Cambridge, MA: MIT Press.

Von Stackelberg, H. 1934/2011. Market structures and equilibrium. Translated by D. Bazin, L. Urch, and R. J. Hill. Reprint, Berlin: Springer-Verlag. 